\title{
Effect of Ca substitution on crystal structure and superconducting properties of ferromagnetic superconductor $\mathrm{RuSr}_{2-x} \mathrm{Ca}_{x} \mathrm{Gd}_{1.4} \mathrm{Ce}_{0.6} \mathrm{Cu}_{2} \mathrm{O}_{10-\delta}$
}

\author{
S. Fallahi ${ }^{\text {a }}$, M. Mazaheri ${ }^{\text {a }}$, N. Nikseresht ${ }^{\mathrm{b}}$, H.M. Rønnow ${ }^{\mathrm{b}}$, M. Akhavan ${ }^{\mathrm{a}, *}$ \\ a Magnet Research Laboratory (MRL), Department of Physics, Sharif University of Technology, P.O. Box 11365-9161, Tehran, Iran \\ b Laboratory for Quantum Magnetism, École Polytechnique Fédérale de Lausanne (EPFL), CH-1015 Lausanne, Switzerland
}

\section{A R T I C L E I N F O}

\section{Article history:}

Received 16 February 2011

Received in revised form

12 September 2011

Available online 20 October 2011

Keywords:

Ruthenocuprate

Magnetism

Superconductivity

Chemical pressure

Hopping exponent

\begin{abstract}
A B S T R A C T
We have investigated the effect of $\mathrm{Ca}$ substitution for $\mathrm{Sr}$ site on structural, magnetic and superconducting properties of $\mathrm{RuSr}_{2-x} \mathrm{Ca}_{x} \mathrm{Gd}_{1.4} \mathrm{Ce}_{0.6} \mathrm{Cu}_{2} \mathrm{O}_{10-\delta}$ system. In this system, the magnetic coupling of $\mathrm{RuO}_{2}$ and $\mathrm{CuO}_{2}$ plays an important role in magnetic and superconducting states. X-ray diffraction analysis shows that all samples are single phase and the lattice parameters decrease continuously by increasing Ca content. The onset superconducting transition temperature is found to decrease with $\mathrm{Ca}$ substitution. As Ca content increases, rotation of the $\mathrm{RuO}_{6}$ octahedron increases and $\mathrm{Ru}-\mathrm{O}(1)-\mathrm{Ru}$ angle decreases. These variations strengthen the magnetic moments in the $\mathrm{RuO}_{2}$ planes. The enhancement of weak ferromagnetic component and hole trapping by Ru magnetic moments in $\mathrm{RuO}_{2}$ planes reduces the electrical conduction, and destroys the superconducting state in the system. Analysis of the resistivity data $(\rho)$ based on the hoping conduction mechanism, indicates a variation of the hoping exponent $(p)$ across the magnetic transition at $T_{m}$. The hoping exponent $p$ is not affected sharply by Ca concentration.
\end{abstract} (c) 2011 Elsevier B.V. All rights reserved.

\section{Introduction}

The coexistence of superconductivity (SC) and ferromagnetism (FM) at microscopic level in the ruthenocuprate family $\mathrm{RuSr}_{2} \mathrm{Gd}_{1.4} \mathrm{Ce}_{0.6} \mathrm{Cu}_{2} \mathrm{O}_{10-\delta}(\mathrm{Ru}-1222)$ is still a controversial subject, and has attracted intense research interest due to the antagonistic nature of SC and FM. The Ru-1222 compound exhibits a weak FM order at relatively high temperature, $T_{M}=180 \mathrm{~K}$, and becomes superconductive at $T_{c}=42 \mathrm{~K}$, within the ferromagnetic order state [1-4]. The weak ferromagnetic component is due to the slight deviations of the magnetic moments of $\mathrm{Ru}$ ions whose magnetic order is predominately antiferromagnetic, from the direction of the $c$-axis caused by antisymmetric Dzyaloshinsky-Moriya (DM) exchange interaction between the neighboring $\mathrm{Ru}$ ions $[1,5]$. The crystal structure of $\mathrm{Ru}-1222$ is similar to that of $\mathrm{REBa}_{2} \mathrm{Cu}_{3} \mathrm{O}_{7-\delta}$ (RE-123), where $\mathrm{Ba}$ and $\mathrm{Cu}$ (chain atom) are replaced by $\mathrm{Sr}$ and $\mathrm{Ru}$, respectively and a three layer fluorite-type block $\mathrm{Gd}(\mathrm{Ce}) \mathrm{O}_{2}$ instead of a single oxygen free rare earth element layer is inserted between the two $\mathrm{CuO}_{2}$ planes of the RE-123 structure [6,7]. The fluorite-type layer supports variable oxygen occupancy, which is denoted with the parameter $\delta$ in the formula. The superconductivity is associated with the $\mathrm{CuO}_{2}$ layers, as in the RE-123 superconductors, while the ferromagnetism arises in the $\mathrm{RuO}_{2}$ layers.

\footnotetext{
* Corresponding author: Tel.: +9821 66164510; fax: +982166022711.

E-mail address: akhavan@sharif.edu (M. Akhavan).
}

There have been many publications in the field of competition between superconductivity and magnetism in the Ru-1222 compound. Studies in this area can be categorized into two basic approaches. The first one measures the physical properties of a standard sample using different techniques, which should supply complementary information sufficient to support or discard a specific model. Significant results have been obtained in this way, although sometimes there have been contradictions in results between different groups [8-12]. A second approach is the investigation of chemically altered compounds, where by proper chemical substitutions one can control relevant parameters that affect the sample's properties and, in this way, get a better understanding of the mechanisms involved in it [13-20]. This second approach is particularly interesting for exploring the possible microscopic coexistence of superconductivity and magnetic order in the ruthenocuprate family.

One strategy to explore the coexistence of SC and FM in $\mathrm{Ru}-1222$ compound is the study of relationship between crystal structure and physical properties of these ferromagnetic superconductors by careful chemical substitutions. Heterovalent substitutions by Sb [18], Pb [19], Sn [20], Mo [13] or Co [14] into Ru site have been used to investigate both magnetic order and superconductivity via the alternations in carrier density in the $\mathrm{CuO}_{2}$ planes and the magnetic coupling between the $\mathrm{Ru}$ ions. However, these heterovalent substitutions have several interconnected effects like valence change, magnetic impurity ion scattering, charge localization, and increase of structural distortions and 
disorder at the same time; it is not useful to investigate the bare relationship between the crystal structure and superconducting properties. From computational point of view, we have recently investigated this relationship between the crystal dimension and electronic properties, in Ru-1212 by applying hydrostatic pressure [21]. The control of crystal structure and physical properties by Ca substitution for $\mathrm{Sr}$, in particular, is very attractive since in this case both ions have similar valence but different ionic radii, with Ca smaller than Sr. This causes no change in the carrier density but due to the different ionic radii, it leads to the compression of lattice along the $c$ direction. By this way, we can tune the separation between the layers in which superconductivity and magnetism occur, retaining the formal oxidation state of the ions. Therefore, it is expected that changes in the superconducting behavior will be mostly due to the possible coupling of magnetism and superconductivity in this system.

In this paper, we have investigated the $\mathrm{Ca}$ substitution in $\mathrm{RuSr}_{2-x} \mathrm{Ca}_{x} \mathrm{Gd}_{1.4} \mathrm{Ce}_{0.6} \mathrm{Cu}_{2} \mathrm{O}_{10-\delta}$ ruthenocuprates, with $x=0,0.1$, $0.2,0.3$. The smaller size of the $\mathrm{Ca}$ ion with respect to the $\mathrm{Sr}$ ion, enables the possibility to change the interlayer separation in which SC and FM occurs, retaining the formal oxidation state of the ions, and to examine the relationship between crystal dimensions and physical properties such as interaction between magnetism and superconductivity in these ferromagnetic superconductors.

\section{Experiments}

The compounds with nominal compositions of $\mathrm{RuSr}_{2-x} \mathrm{Ca}_{x}$ $\mathrm{Gd}_{1.4} \mathrm{Ce}_{0.6} \mathrm{Cu}_{2} \mathrm{O}_{10-\delta}$ with $x=0,0.1,0.2,0.3$ were prepared by the solid state reaction method from starting powders $\mathrm{RuO}_{2}, \mathrm{SrCO}_{3}$, $\mathrm{CaCO}_{3}, \mathrm{Gd}_{2} \mathrm{O}_{3}, \mathrm{CeO}_{2}$ and $\mathrm{CuO}$ with $99.9 \%$ purity. The powders were ground, die-pressed into pellets and calcined for $24 \mathrm{~h}$ at $950{ }^{\circ} \mathrm{C}$ in air. The calcination was repeated several times with intermediate grinding to reach a homogeneous powder. The samples were reground, re-pelleted and sintered for $72 \mathrm{~h}$ in oxygen atmosphere at $1070{ }^{\circ} \mathrm{C}$ and then slowly cooled down to ambient temperature. The phase purity and the lattice parameters examined by a Philips Xpert powder diffractometer with $\mathrm{CuK} \alpha$ radiation. The magnetization measurements performed using SQUID magnetometer. Zero field cooled (ZFC) and field cooled (FC) measurements performed at 50 Oe field in the range $2-200 \mathrm{~K}$. An ac four-probe method with $f=33 \mathrm{~Hz}$ used for the resistivity measurements of the samples from 10 to $300 \mathrm{~K}$. The measuring current for the resistivity was $10 \mathrm{~mA}$. A Lake Shore-330 temperature controller with a Pt-100 resistor used to indicate and control the temperature.

\section{Results and discussion}

\subsection{XRD and structural analysis}

Fig. 1 shows the X-ray diffraction (XRD) patterns in the range from $20^{\circ}$ to $100^{\circ}$ for $\mathrm{RuSr}_{2-x} \mathrm{Ca}_{x} \mathrm{Gd}_{1.4} \mathrm{Ce}_{0.6} \mathrm{Cu}_{2} \mathrm{O}_{10-\delta}$ samples with $x=0,0.1,0.2,0.3$, respectively. Structural analysis was carried out using general structure analysis system GSAS for Rietveld refinement of X-ray diffraction spectra [22]. X-ray diffraction analysis shows that all samples are single phase with no trace of impurity phases. The diffraction peaks were successfully indexed on the basis of a tetragonal unit cell with the I4/MMM (No. 139) space group (Table 1), as denoted in Fig. 1.

Upon Ca substitution, the overall Bragg reflections shift toward the high-angle sides. From Rietveld refinement analysis, the unit cell volume is found to slightly decrease on Ca substitution. The lattice parameters $a$ and $c$ for the samples as a function of $\mathrm{Ca}$ content are presented in Fig. 2. This result shows that both lattice

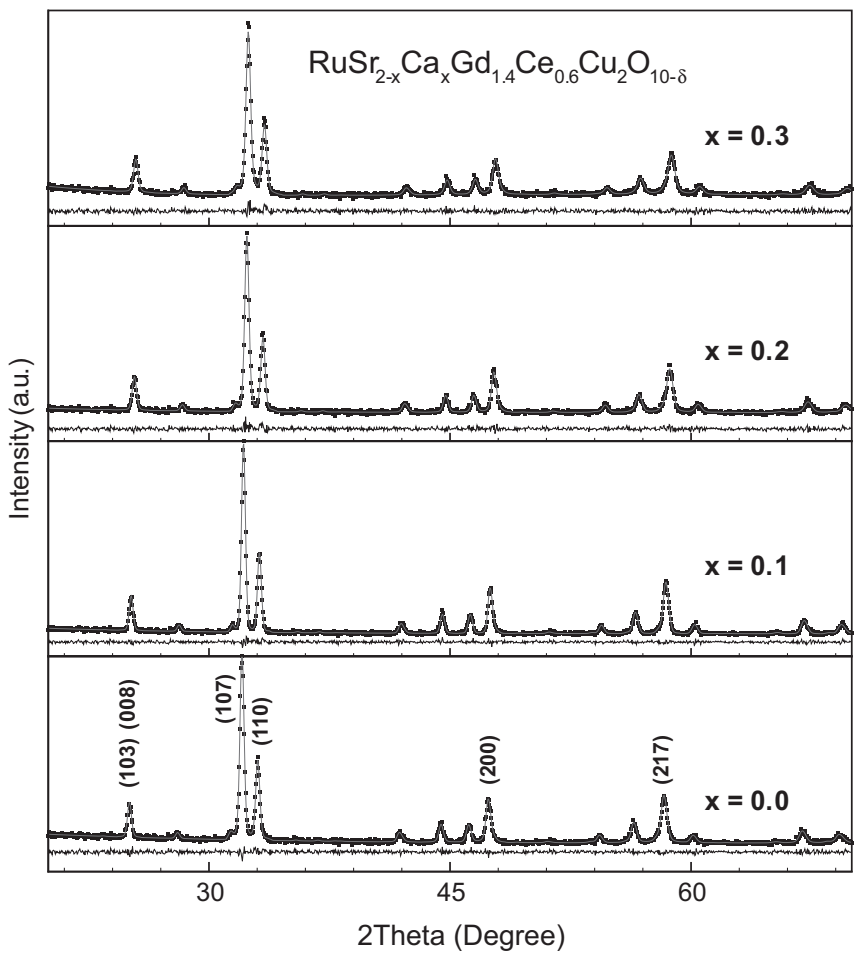

Fig. 1. Powder XRD patterns of the Ca substituted $\mathrm{RuSr}_{2-x} \mathrm{Ca}_{x} \mathrm{Gd}_{1.4} \mathrm{Ce}_{0.6} \mathrm{Cu}_{2} \mathrm{O}_{10-\delta}$.

Table 1

Results of refined atomic parameters for $\mathrm{RuSr}_{2-x} \mathrm{Ca}_{x} \mathrm{Gd}_{14} \mathrm{Ce}_{06} \mathrm{Cu}_{2} \mathrm{O}_{10-\delta}$ solid solutions using GSAS program. The structure model with the I4/MMM (No. 139) space group yields the best fit for all samples. Atom positions are Ru 2a $(0,0,0)$ $\mathrm{Sr} / \mathrm{Ca} 4 \mathrm{e}(0,0, z), \mathrm{Gd} / \mathrm{Ce} 4 \mathrm{e}(0,0, z), \mathrm{Cu} 4 \mathrm{e}(0,0, z), \mathrm{O}(1) 8 \mathrm{j}\left(\frac{1}{2}, y, 0\right), \mathrm{O}(2) 4 \mathrm{e}(0,0, z)$, $\mathrm{O}(3) 8 \mathrm{~g}\left(0, \frac{1}{2}, z\right), \mathrm{O}(4) 4 \mathrm{~d}\left(0, \frac{1}{2}, \frac{1}{4}\right)$.

\begin{tabular}{llllll}
\hline Atom & Parameter & $x=0$ & $x=0.1$ & $x=0.2$ & $x=0.3$ \\
\hline & $a(\AA)$ & $3.8431(3)$ & $3.8402(2)$ & $3.8371(3)$ & $3.8359(4)$ \\
& $c(\AA)$ & $28.587(2)$ & $28.582(2)$ & $28.573(2)$ & $28.569(2)$ \\
$\mathrm{Ru}$ & $U_{\text {iso }}(\AA)^{2}$ & $0.009(2)$ & $0.011(2)$ & $0.008(3)$ & $0.010(2)$ \\
$\mathrm{Sr} / \mathrm{Ca}$ & $z$ & $0.4219(3)$ & $0.4216(2)$ & $0.4211(2)$ & $0.4209(3)$ \\
& $U_{\text {iso }}(\AA)^{2}$ & $0.019(2)$ & $0.021(3)$ & $0.019(3)$ & $0.022(2)$ \\
$\mathrm{Gd} / \mathrm{Ce}$ & $z$ & $0.2945(2)$ & $0.2943(2)$ & $0.2939(3)$ & $0.2935(2)$ \\
& $U_{\text {iso }}(\AA)^{2}$ & $0.012(1)$ & $0.011(1)$ & $0.011(1)$ & $0.010(1)$ \\
$\mathrm{Cu}$ & $z$ & $0.1411(3)$ & $0.1403(2)$ & $0.1396(3)$ & $0.1391(3)$ \\
& $U_{\text {iso }}(\AA)^{2}$ & $0.021(1)$ & $0.021(2)$ & $0.020(2)$ & $0.019(2)$ \\
$\mathrm{O}(1)$ & $y$ & $0.1184(5)$ & $0.1315(4)$ & $0.1422(4)$ & $0.1508(5)$ \\
& $U_{\text {iso }}(\AA)^{2}$ & $0.021(2)$ & $0.022(2)$ & $0.022(1)$ & $0.020(2)$ \\
$\mathrm{O}(2)$ & $z$ & $0.0672(3)$ & $0.0643(2)$ & $0.0617(3)$ & $0.0597(3)$ \\
& $U_{\text {iso }}(\AA)^{2}$ & $0.035(3)$ & $0.036(3)$ & $0.035(3)$ & $0.038(3)$ \\
$\mathrm{O}(3)$ & $z$ & $0.1492(2)$ & $0.1464(2)$ & $0.1431(3)$ & $0.1424(3)$ \\
& $U_{\text {iso }}(\AA)^{2}$ & $0.038(3)$ & $0.036(3)$ & $0.039(3)$ & $0.039(3)$ \\
$\mathrm{O}(4)$ & $U_{\text {iso }}(\AA)^{2}$ & $0.037(3)$ & $0.039(3)$ & $0.037(3)$ & $0.038(3)$ \\
\end{tabular}

parameters tend to decrease by increasing the Ca-doping concentration in the single-phase region of $0.0 \leq x \leq 0.3$, indicating that $\mathrm{Ca}$ entered the lattice, which is in good agreement with the smaller ionic size of $\mathrm{Ca}^{2+}=1.48 \AA$ with respect to $\mathrm{Sr}^{2+}=1.58 \AA$ with 12-fold coordination number. A mismatch between the inplane $\mathrm{Ru}-\mathrm{O}$ and $\mathrm{Cu}-\mathrm{O}$ bonds leads to the rotations of the $\mathrm{RuO}_{6}$ octahedra around the $c$-axis. From the refined crystal structure, we have extracted the bond lengths and the rotation angles for the $\mathrm{RuO}_{6}$ octahedra and the buckling angle in the $\mathrm{Cu}-\mathrm{O}$ planes. The reduction in the $a$ cell parameter, on going from $x=0.0$ to 0.3 in $\mathrm{RuSr}_{2-x} \mathrm{Ca}_{x} \mathrm{Gd}_{1.4} \mathrm{Ce}_{0.6} \mathrm{Cu}_{2} \mathrm{O}_{10-\delta}$ increases the rotations of the $\mathrm{RuO}_{6}$ octahedra, quantified by the $\mathrm{Ru}-\mathrm{O}(1)-\mathrm{Ru}$ angle in Table 2 . 
We found that $\mathrm{Ca}$ substitution for $\mathrm{Sr}$ enhances the structure distortion. The rotation angle of $\mathrm{RuO}_{6}$ octahedron, as is shown in Table 2, increases from $13.7^{\circ}$ for $x=0.0-17.1^{\circ}$ for $x=0.3$.

The out of plane $\mathrm{Cu}-\mathrm{O}(3)$ bond length decreases with $\mathrm{Ca}$ substitution. This variation is greater than the error range for the calculated bond length $(\sigma= \pm 0.001 \AA)$. The present work highlights that the unit cell dimension of Ru-1222 along the $c$ axis can be adjusted to decrease by isovalent Ca substitution for $\mathrm{Sr}$, as estimated from Fig. 2. It must be noted that Ca substitution induces prominent change in the $\mathrm{Cu}-\mathrm{O}(3)$ bond distance. This may be due to cation disorder between $\mathrm{Cu}$ and $\mathrm{Ru}$ and/or between Ca and Gd sites in the refined occupancy of these ions.

\section{DC magnetism}

The temperature dependence of magnetization for all samples is shown in Fig. 3. The samples were cooled in zero magnetic field down to the lowest accessible temperature (2 K). After temperature stabilization, a magnetic field of 50 Oe was applied and the

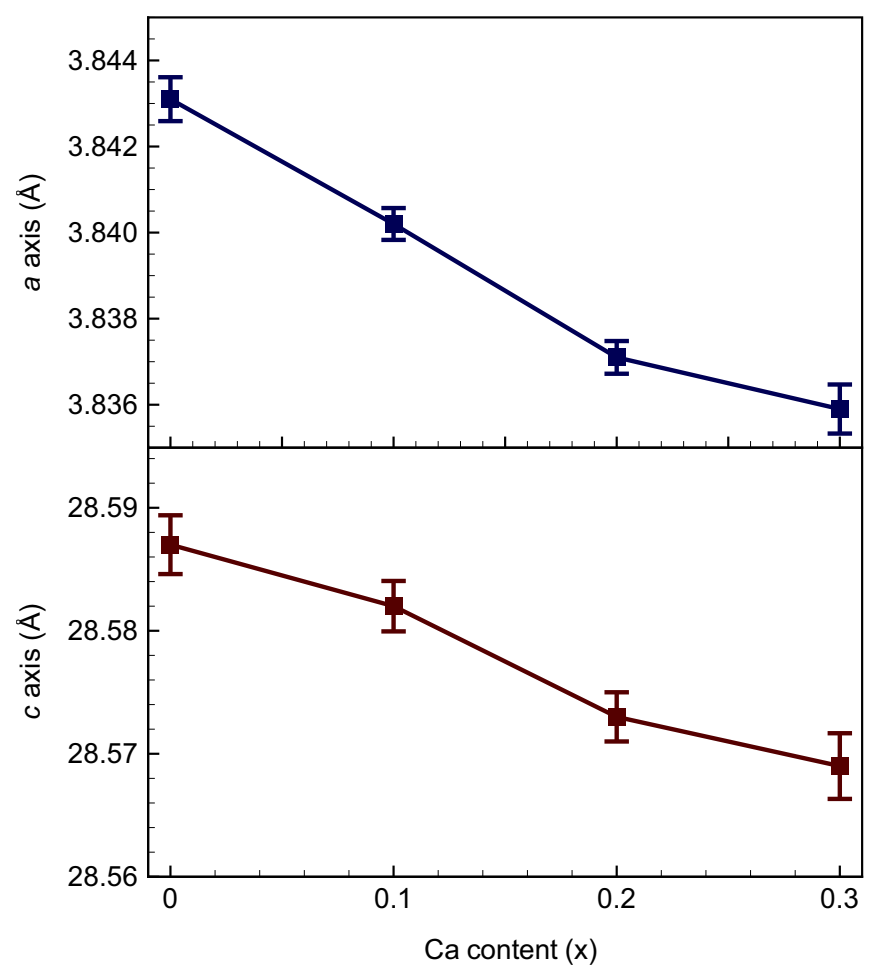

Fig. 2. Variations of $a$ and $c$ lattice parameters with Ca-doping content, $x$ for $\mathrm{RuSr}_{2-x} \mathrm{Ca}_{x} \mathrm{Gd}_{1.4} \mathrm{Ce}_{0.6} \mathrm{Cu}_{2} \mathrm{O}_{10-\delta}$ samples.
ZFC curve measured up to $160 \mathrm{~K}$. The FC data obtained while the temperature was decreased back to $2 \mathrm{~K}$, keeping the same applied magnetic field. In all samples, the ZFC curves fall below the FC ones. We observe that the overall shape of the magnetization curve for all substituted samples is similar to the result obtained for the pristine compound, although some important differences between these curves can be pointed out.

There are two characteristic temperatures: $T_{m}$ and $T_{i r r}$ with $T_{i r r}<T_{m}$ in the temperature dependence of the dc magnetization for the synthesized samples. We define $T_{i r r}$ as the temperature below which the magnetization becomes strongly hysteretic, which can be interpreted as weak ferromagnetism resulting from canting of the Ru magnetic moment. The upper characteristic temperature, $T_{m}$, which marks the initial increase of the magnetization, does not depend on the amount of Ca content. On the other hand, $T_{i r r}$ which reflects the beginning of ferromagnetic transition temperature, increases by Ca concentration. This is due to the decrease in the $\mathrm{Ru}-\mathrm{O}(1)$ bond length and enhancement of the $\mathrm{RuO}_{6}$ octahedron rotations which strengthens the DM interaction between the nearest Ru moments. There is no evidence of bulk superconductivity our synthesized samples, which indicate the screening of the superconducting grains caused by the applied magnetic field.

The isothermal $\mathrm{M}(\mathrm{H})$ measurements have been carried out in the range $-6 \mathrm{~T}$ to $6 \mathrm{~T}$ at $5 \mathrm{~K}$, and the results obtained for the $x=0.0$ and $x=0.3$ samples are shown in Fig. 4 . The curves show a slowly saturating curve, which is well described by free $J=3 / 2$ spins, and a second component showing classical hysteresis behavior.

Quantitative analysis of the XANES spectra of Ru-1222 material revealed that the $\mathrm{Ru}$ valance is between $\mathrm{Ru}^{+4} / \mathrm{Ru}^{+5}$ which, in case of Ru-1222 materials it is closer to +5 [23]. The electronic configuration of $\mathrm{Ru}^{+5}$ is $t_{2 g}^{3}$. So it can have high spin state $(S=3 / 2)$ with the theoretical magnetic moment value of $3 \mu_{B} / \mathrm{Ru}$ or, low spin state $(S=1 / 2)$ with the theoretical magnetic moment value of $1 \mu_{B} / \mathrm{Ru}$. Our refined fitting parameters confirm the high spin state value. The red line in the right panel, is the fit to Brillouin function to subtract the paramagnetic part. I used $J=3 / 2$ and $T=5 \mathrm{~K}$ as fitting parameters

$B_{J}(x)=\frac{2 J+1}{2 J} \operatorname{coth}\left(\frac{2 J+1}{2 J} x\right)-\frac{1}{2 J} \operatorname{coth}\left(\frac{1}{2 J} x\right)$,

$M=N g \mu_{B} J \cdot B_{J}(x)$, where $x=\beta g \mu_{B} J H$.

By fitting the hysteresis data to the Brillouin function, we could manage to subtract the paramagnetic contribution. The right panel of each graph below is the ferromagnetic feature remained after paramagnetic part subtraction. There is a slight increase in the $M_{\text {sat }}$ associated within the Ru sublattice as seen in Fig. 4 which is related to the increase of the localized magnetic moment as

Table 2

Calculated interatomic distances in $\AA$ and bond angles $\left(^{\circ}\right.$ ) for $\mathrm{RuSr}_{2-x} \mathrm{Ca}_{x} \mathrm{Gd}_{1.4} \mathrm{Ce}_{0.6} \mathrm{Cu}_{2} \mathrm{O}_{10-\delta}$ for $x=0.0,0.1,0.2$ and 0.3 .

\begin{tabular}{lllll}
\hline Bond & $x=0$ & $x=0.1$ & $x=0.2$ & $x=0.3$ \\
\hline $\mathrm{Ru}-\mathrm{O}(1)$ & $1.9759(3)$ & $1.9969(2)$ & $1.9946(3)$ & $2.0048(3)$ \\
$\mathrm{Ru}-\mathrm{O}(2)$ & $1.890(1)$ & $1.848(5)$ & $1.793(5)$ & $1.696(6)$ \\
$\mathrm{Sr}-\mathrm{O}(1)$ & $2.69(1) / 3.28(1)$ & $2.620(4) / 3.325(3)$ & $2.614(5) / 3.319(3)$ & $2.591(5) / 3.341(4)$ \\
$\mathrm{Sr}-\mathrm{O}(2)$ & $2.745(4)$ & $2.743(2)$ & $2.747(3)$ & $2.764(4)$ \\
$\mathrm{Sr}-\mathrm{O}(3)$ & $2.753(5)$ & $2.748(6)$ & $2.697(6)$ & $2.659(7)$ \\
$\mathrm{Cu}-\mathrm{O}(2)$ & $2.1124(5)$ & $2.1720(3)$ & $2.2258(3)$ & $2.2672(4)$ \\
$\mathrm{Cu}-\mathrm{O}(3)$ & $1.937(1)$ & $1.929(1)$ & $1.921(1)$ & $1.919(1)$ \\
$\mathrm{Gd} / \mathrm{Ce}-\mathrm{O}(3)$ & $2.548(5)$ & $2.547(6)$ & $2.590(5)$ & $2.630(4)$ \\
$\mathrm{Gd} / \mathrm{Ce}-\mathrm{O}(4)$ & $2.288(6)$ & $2.307(5)$ & $2.309(4)$ & $1.309(5)$ \\
$\mathrm{Cu}-\mathrm{O}(3)-\mathrm{Cu}$ & $166.3(1)$ & $169.6(2)$ & $174.1(2)$ & $174.4(2)$ \\
$\mathrm{Ru}-\mathrm{O}(1)-\mathrm{Ru}$ & $153.4(1)$ & $150.5(2)$ & $148.2(2)$ & $146.4(2)$ \\
\hline
\end{tabular}



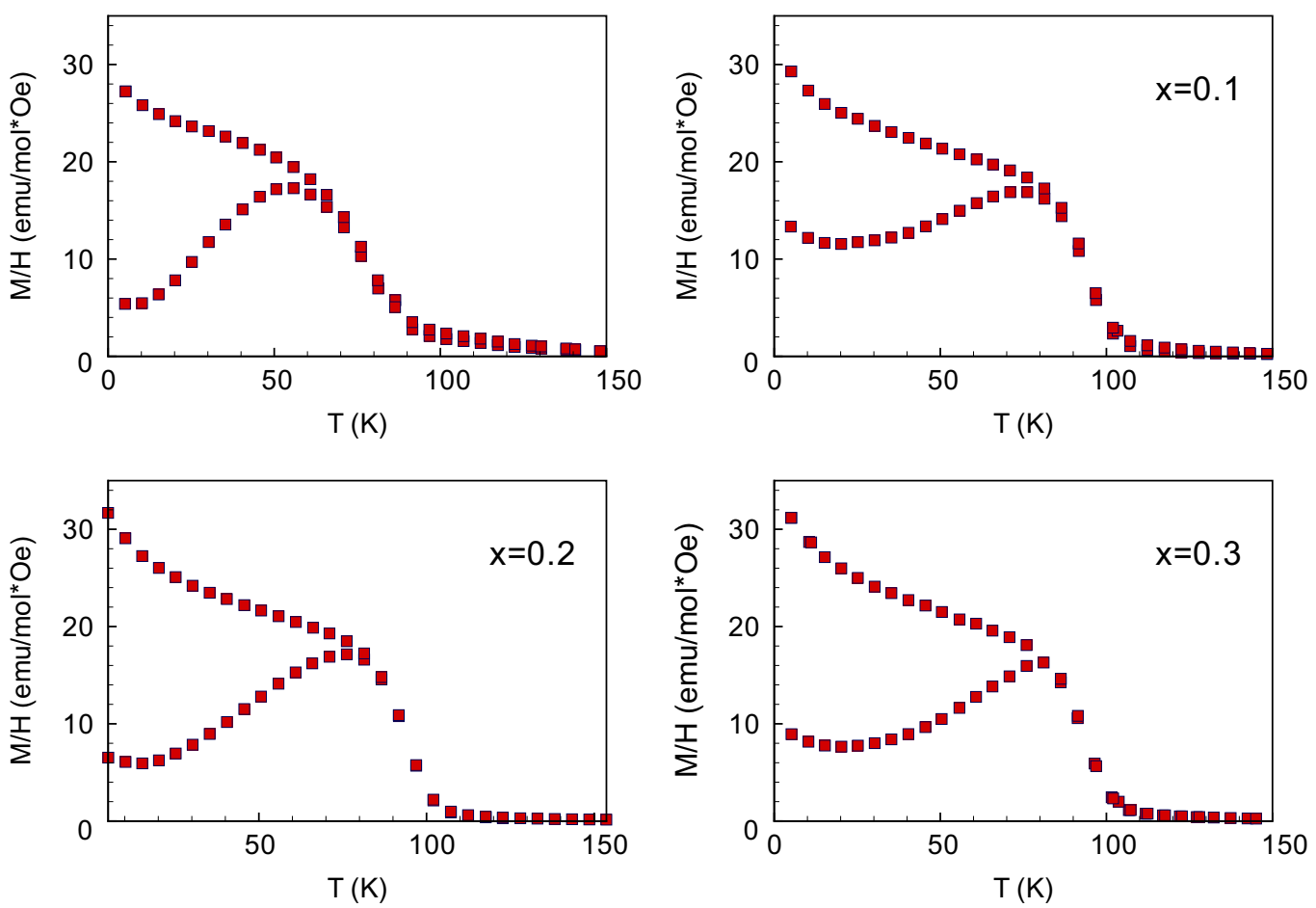

Fig. 3. Temperature dependence of FC and ZFC magnetization for $\mathrm{RuSr}_{2-x} \mathrm{Ca}_{x} \mathrm{Gd}_{1.4} \mathrm{Ce}_{0.6} \mathrm{Cu}_{2} \mathrm{O}_{10-\delta}$ samples.

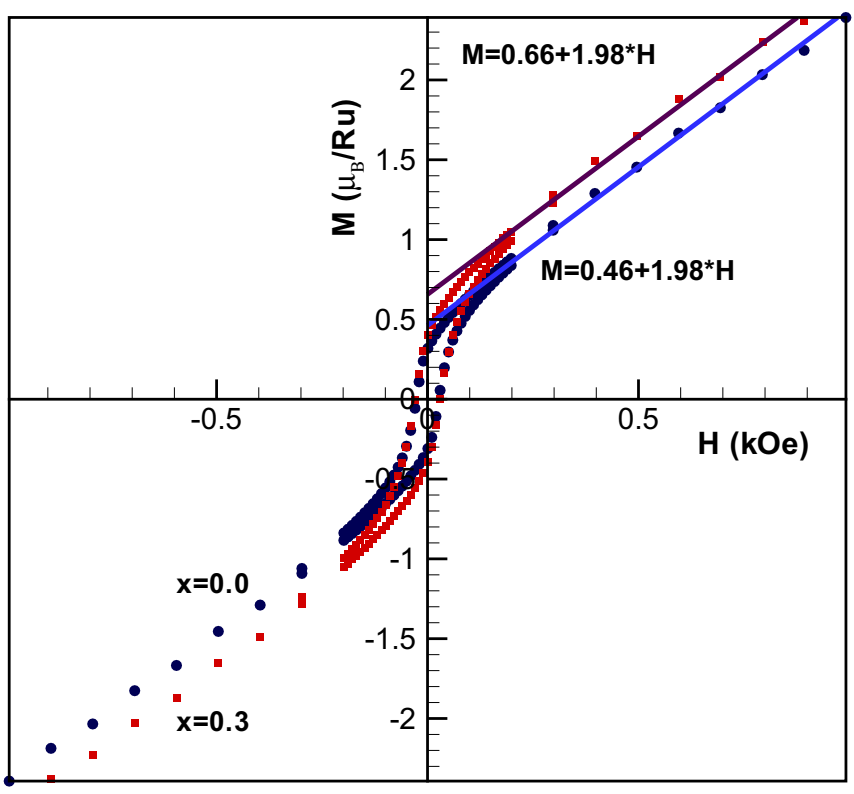

Fig. 4. Hysteresis field dependence of magnetization for $\mathrm{RuSr}_{2-x} \mathrm{Ca}_{x} \mathrm{Gd}_{1.4} \mathrm{Ce}_{0.6}$ $\mathrm{Cu}_{2} \mathrm{O}_{10-\delta}$ samples. (For interpretation of the references to color in this figure legend, the reader is referred to the web version of this article.)

a result of $\mathrm{RuO}_{6}$ octahedra rotations which causes band narrowing. The results of the ac susceptibility measurements are presented in Fig. 5. From the figure it is seen that for $x=0.0$ there are two peaks corresponding to magnetic ordering, a small one at higher temperature of the order of $120 \mathrm{~K}$ and a dominant one around $95 \mathrm{~K}$. The smaller magnetic peak is due to the possible antisymmetric exchange coupling of the DM type between neighboring Ru moments caused by a local distortion that breaks the tetragonal symmetry of the $\mathrm{RuO}_{6}$ octahedra. Due to this interaction the field causes the adjacent spins to cant slightly out of their original direction thereby lending a component in the direction of the applied field.

\section{Electrical resistivity}

The effect of Ca substitutions in the Sr site on the temperature dependence of the electrical resistivity is shown in Fig. 6. The inset shows the room temperature resistivity $\rho(300)$ and the onset superconducting transition temperature $\left(T_{c}^{\text {onset }}\right)$ of the samples. By increasing the Ca-doping content, $\rho(300)$ increases while $T_{c}$ decreases, which may be due to the disorder-induced localization.

The distortion of the $\mathrm{RuO}_{6}$ octahedra due to Ca substitution for $\mathrm{Sr}$ might lead to strong narrowing of the bands which results in localization of the carriers. Hence the transport behavior and the superconducting transition temperature drop with the $\mathrm{Ca}$ concentration. XRD analysis also confirmed that Ca substitution introduces more distortion in the $\mathrm{RuO}_{6}$ octahedra and together with the aforementioned cationic disorder, cause more magnetic scattering or trapping, that prevents the movement of hole carriers and results in hole localization.

The bond valence sum concept for cuprates suggests a slight decrease of the $\mathrm{Cu}-\mathrm{O}(3)$ bond distance by $\mathrm{Ca}$ content, which implies a minute increase in the hole density. This is in contrast with the observed weakening of the metallic behavior in the samples by Ca substitution. It might be a result of cationic disorder which localizes the hole carrier. Although the origin of the change in $T_{c}^{\text {onset }}$ caused by Ca substitution is not very clear, it seems to be related to the enhancement of structural disorder such as cation mixing, as found from the XRD analysis.

Taking into account the fact that the structural disordered sites can trap hole carriers in the $\mathrm{CuO}_{2}$ layer, the structural disorder could be a reason for the depression of superconductivity in the Ca substituted samples.

From the above analysis, we conclude that Ca substitution not only changes the microstructure, but also introduces disorder to the $\mathrm{RuSr}_{2-x} \mathrm{Ca}_{x} \mathrm{Gd}_{1.4} \mathrm{Ce}_{0.6} \mathrm{Cu}_{2} \mathrm{O}_{10-\delta}$ system, which results in the increase of normal resistivity. In addition to the disorder induced localization, there might exist magnetic trapping or scattering of the holes due to the presence of magnetic fluctuation. 

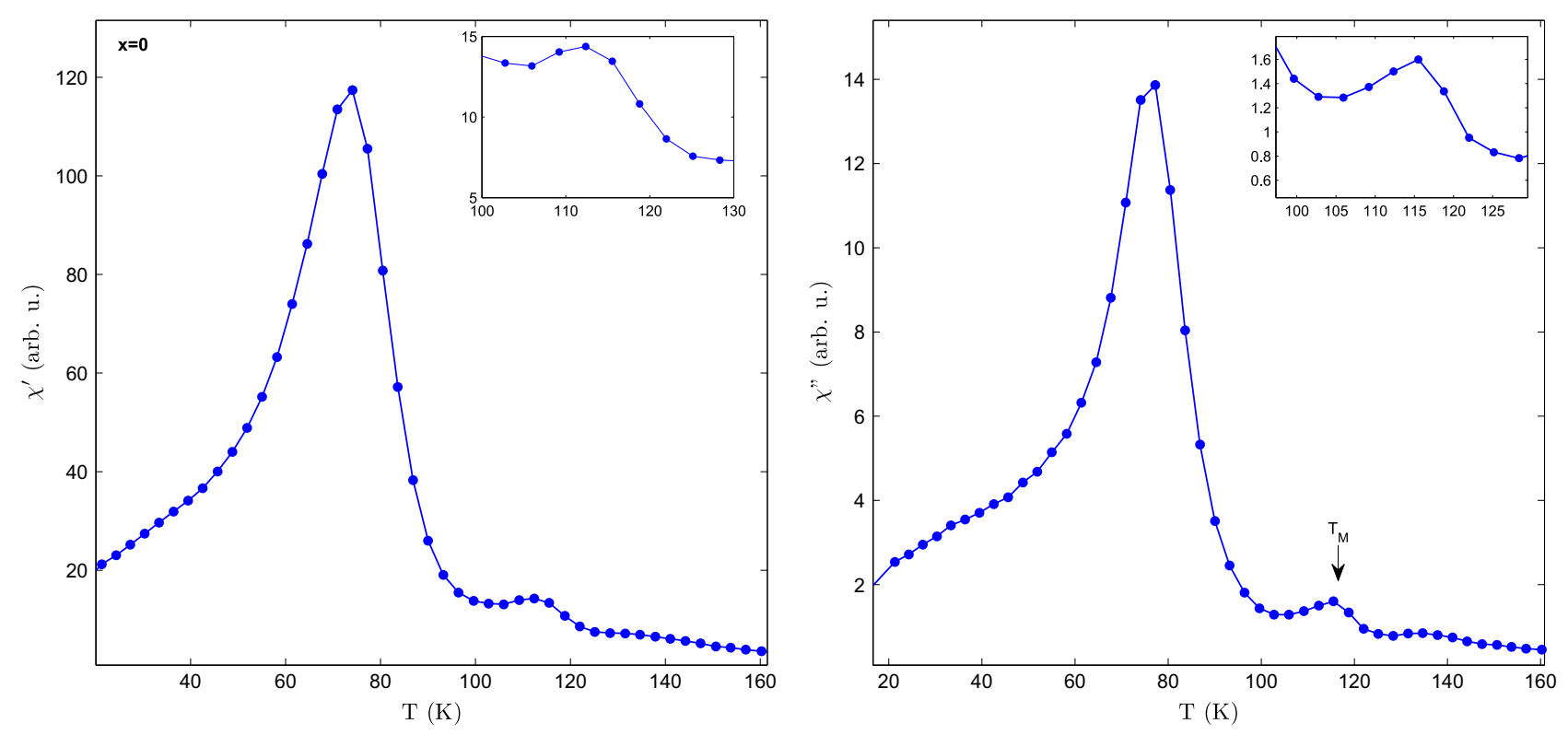

Fig. 5. Real and imaginary components of the AC susceptibility for $x=0$, with an inset zooming into the two small peaks in $\chi^{\prime \prime}$.

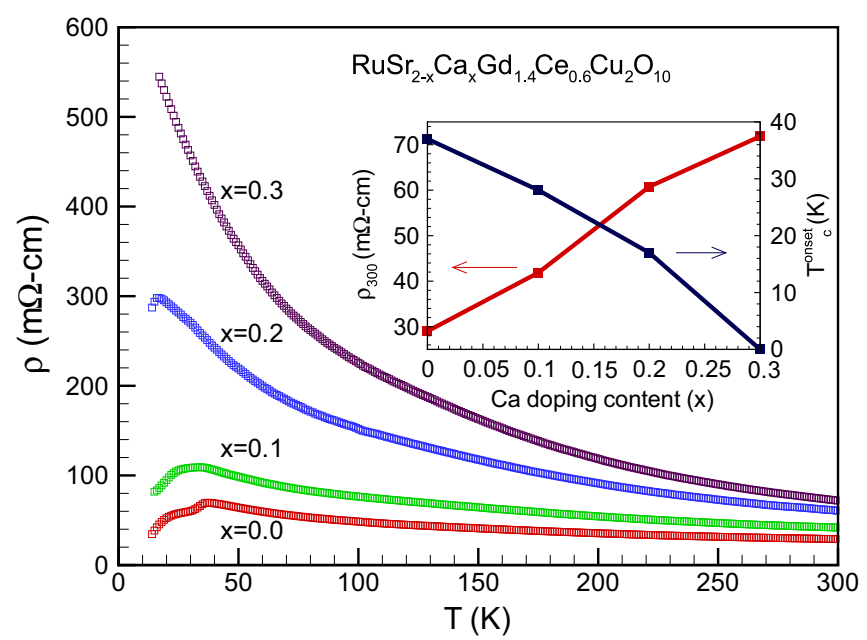

Fig. 6. Temperature dependence of electrical resistivity for $\mathrm{RuSr}_{2-x} \mathrm{Ca}_{x} \mathrm{Gd}_{1.4} \mathrm{Ce}_{0.6}$ $\mathrm{Cu}_{2} \mathrm{O}_{10-\delta}$ samples.

A careful study of the normal state properties of Ca substituted ruthenocuprate based on hopping conduction analysis, indicates a transitional behavior of hopping conduction at the magnetic transition. In fact the correlation between the electrical transport and magnetic order properties could improve our understanding of superconductivity and reveal the connection between the superconductivity and magnetic states with the normal state characteristic.

The temperature dependent resistivity of hopping conduction can be expressed by the following equation [24]:

$\rho(T)=\rho_{0} \exp \left(\frac{2 r}{\alpha}+\frac{\varepsilon}{k_{B} T}\right)$,

where $\rho_{0}$ depends on particular physical and material properties of samples, $r$ is the hopping distance, $\varepsilon$ is the hopping energy, $\alpha$ is the localization length, and $k_{B}$ is the Boltzmann constant. The hopping distance and hopping energy obey a constraint condition

$$
\left(\frac{4 \pi}{3}\right) r^{3} \int g\left(\varepsilon^{\prime}\right) d \varepsilon^{\prime}=1
$$

where $g(\varepsilon)$ is the Local Density of State (LDOS). Under this condition, the most probable hopping distance and hopping energy are determined by minimizing the term $2 r / \alpha+\varepsilon / k_{B} T$ in Eq. (3). The different distribution of LDOS will lead to different formula for the temperature dependent resistivity. For example (I) a uniform LDOS near Fermi surface corresponds to Mott's variable range hopping (VRH) model [25]; $\rho(\mathrm{T}) \propto \exp \left(T_{0} / T\right)^{0.25}$, where $T_{0}$ is a characteristic temperature. (II) Taking the electronic Coulomb interaction into account, Efros and Shklovskii (ES) deduced a parabolic distribution of LDOS near Fermi level and obtained $\rho(T) \propto \exp \left(T_{0} / T\right)^{0.5}$. In general, the temperature dependent resistivity of the system with hopping conduction can be described by the following:

$\rho(T) \propto \exp \left(T_{0} / T\right)^{p}$.

By using the method proposed by Shklovskii and Efros, $p$ can be determined from the slope of a plot of $\ln (\mathrm{W})$ versus $\ln (\mathrm{T})$ as follows:

$W=\frac{1}{T} \frac{d(\ln \rho)}{d\left(T^{-1}\right)} \approx p\left(\frac{T_{0}}{T}\right)^{p}$,

Fig. 7 indicates that $p$ is divided into two different ranges around $T_{M}$. the value is close to 0.25 below $T_{M}$ and changes to 0.5 above $T_{M}$, which indicates a parabolic like distribution of LDOS in the system. The variation in the hopping exponent is caused by a change of the distribution of LDOS near Fermi level [26,27]. The distribution of the localized electronic states closely links to the degree of disorder in the system. It has been already reported in mixed valance manganites with perovskite structure [28] that spatial fluctuation of spin-dependent potentials related to the magnetic order lead to the localization of electronic states.

In our synthesized compounds, the spin-dependent potential may vary across the magnetic transition, which causes the change of distribution in LDOS, and leads to the variation in the hopping exponent. It should be noted here that the hopping exponent does not vary sharply by Ca doping, which can be concluded as decoupling of superconducting and magnetic layers in the studied range concentrations. In a separate analysis of hopping conduction below magnetic transition, we found that the localization length $\alpha$ decreases with the increasing $x$. This implies that the degree of hole localization is enhanced with the increase in disorder as derived from the XRD analysis. 

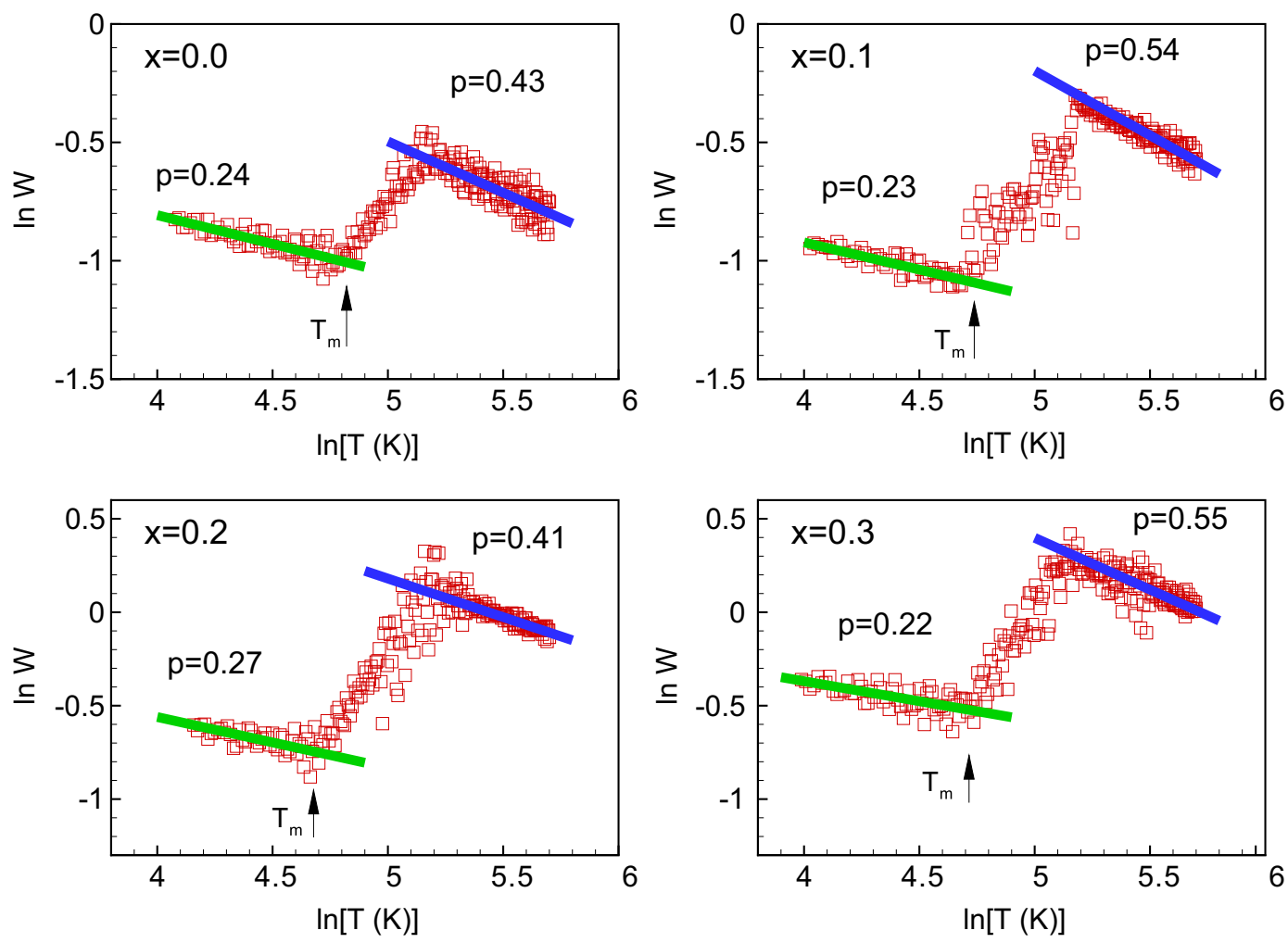

Fig. 7. Variation of hopping conduction across magnetic transition in $\mathrm{RuSr}_{2-x} \mathrm{Ca}_{x} \mathrm{Gd}_{1.4} \mathrm{Ce}_{0.6} \mathrm{Cu}_{2} \mathrm{O}_{10-\delta}$ compounds.

\section{Summary}

We have synthesized the Ca substituted $\mathrm{RuSr}_{2-x} \mathrm{Ca}_{x} \mathrm{Gd}_{1.4} \mathrm{Ce}_{0.6}$ $\mathrm{Cu}_{2} \mathrm{O}_{10-\delta}$ compounds with $x=0-0.3$, which enables us to map out the changes in superconducting and magnetic properties for $\mathrm{Ru}-1222$ as a function of crystal lattice parameters. The XRD analysis revealed that replacement of $\mathrm{Sr}$ with $\mathrm{Ca}$ reduces both the unit cell dimension of Ru-1222 ruthenocuprate along the $c$-axis and the interlayer distance between the $\mathrm{CuO}_{2}$ and $\mathrm{RuO}_{2}$ layers. The magnetic transition temperature decreased by increasing $\mathrm{Ca}$ concentration, while $T_{i r r}$ increased due to enhancement of DM interaction between the Ru magnetic moments. The onset of the superconducting transition temperature $T_{c}^{\text {onset }}$ dropped by $\mathrm{Ca}$ substitution. This could be due to the spatially inhomogeneous magnetic scattering of hole carrier by increasing the local distortion of $\mathrm{RuO}_{6}$ octahedron. The resistivity data analysis based on hopping conduction indicates variation of the hopping exponent $p$ across magnetic transition at $T_{m}$. The hopping exponent $p$ is not affected sharply by Ca substitution. This may be an indication of decoupling of the $\mathrm{RuO}_{2}$ and $\mathrm{CuO}_{2}$ planes at these level of doping, and the prominent mechanism for depression of superconductivity is the magnetic trapping of the hole carriers.

\section{References}

[1] I. Felner, U. Asaf, Y. Levi, O. Millo, Physical Review B 55 (1997) R3374.

[2] W.K. Yeoh, C.A. Kek, R. Abd-Shukor, Superconductor Science and Technology 15 (2002) 361.

[3] L. Bauernfeind, W. Widder, H.F. Braun, Journal of Physics and Chemistry of Solids 254 (1995) 151.

[4] G.V.M. Williams, M. Ryan, Physical Review B 64 (2001) 094515.
[5] J. Dzyaloshinsky, Journal of Physics and Chemistry of Solids 4 (1958) 241.

[6] C.S. Knee, B.D. Rainford, M.T. Weller, Journal of Materials Chemistry 10 (2000) 2445.

[7] A.C. Mclaughlin, J.P. Attfield, Physical Review B 68 (2003) 014503.

[8] I. Felner, E. Galstyan, R.H. Herber, I. Nowik, Physical Review B 70 (2004) 094504.

[9] Y.Y. Xue, D.H. Cao, B. Lorenz, C.W. Chu, Physical Review B 65 (2001) 020511(R).

[10] A. Lopez, I.S. Azevedo, J.L. Gonzalez, E. Baggio-Saitovitch, H. Micklitz, Physica C 442 (2006) 33.

[11] G.V.M. Williams, S. Kramer, Physical Review B 62 (2000) 4132.

[12] A. Butera, A. Fainstein, E. Winkler, J. Tallon, Physical Review B 63 (2001) 054442 .

[13] V.P.S. Awana, R. Lal, H. Kishan, A.V. Narlikar, M. Peurla, R. Laiho, Physical Review B 73 (2006) 014517.

[14] V.P.S. Awana, H. Kishan, O. Eshkenazi, I. Felner, R. Rawat, V. Ganesan, A.V. Narlikar, Journal of Physics: Condensed Matter 19 (2007) 026203.

[15] I. Felner, E. Galstyan, International Journal of Modern Physics B 17 (2003) 3617.

[16] H.K. Lee, Y.C. Kim, International Journal of Modern Physics B 17 (2003) 3682.

[17] H.K. Lee, G.V.M. Williams, Physica C 415 (2004) 172.

[18] L. Shi, G. Li, S.J. Feng, X.-G. Li, Physica Status Solidi A 1 (2003) 137.

[19] L. Shi, G. Li, Y. Pu, X.D. Zhang, S.J. Feng, X.-G. Li, Materials Letters 57 (2003) 3919.

[20] H.K. Lee, H.M. Park, G.V.M. Williams, International Journal of Modern Physics B 19 (2005) 353.

[21] S. Fallahi, H. Hadipour, M. Akhavan, The European Physical Journal B 78 (2010) 531.

[22] A.C. Larson, R.B.V. Dreele, Los Alamos National Laboratory Report LAUR 86 , $1985,748$.

[23] V.P.S. Awana, M. Karppinen, H. Yamauchi, M. Matvejeff, R.S. Liu, L.-Y. Jang, Journal of Low Temperature Physics 131 (2003) 1211.

[24] R. Schmidt, A. Basu, A.W. Brinkman, Physical Review B 72 (2005) 115101.

[25] N.F. Mott, Conduction in Non-Crystalline Materials, , 1993.

[26] S. Nakatsuji, V. Dobrosavljevic, D. Tanaskovic, M. Minakata, H. Fukazawa Y. Maeno, Physical Review Letters 93 (2004) 146401.

[27] Y. Meir, Physical Review Letters 77 (1996) 5265.

[28] J. Wu, Z. Huang, Y. Hou, Y. Gao, J. Chu, Applied Physics Letters 96 (2010) 082103. 